\title{
Unfamiliar faces are not faces: Evidence from a matching task
}

\author{
AHMED M. MEGREYA and A. MIKE BURTON \\ University of Glasgow, Glasgow, Scotland
}

\begin{abstract}
It is difficult to match two images of the same unfamiliar face, even under good conditions. Here, we show that there are large individual differences on unfamiliar face matching. Initially, we tried to predict these using tests of visual short-term memory, cognitive style, and perceptual speed. Moderate correlations were produced by various components of these tests. In three other experiments, we found very strong correlations between face matching and inverted face matching on the same test. Finally, we examined potential associations between familiar and unfamiliar face processing. Strong correlations were found between familiar and unfamiliar face processing, but only when the familiar faces were inverted. We conclude that unfamiliar faces are processed for identity in a qualitatively different way than are familiar faces.
\end{abstract}

People are remarkably poor at matching images of unfamiliar faces. For example, Figures 1 and 2 show arrays, reproduced from Bruce et al. (1999). Subjects were shown arrays of this type and told that the face of the person at the top might or might not be among the 10 targets. They were asked to decide whether or not that face was present and, if so, to pick the correct target. ${ }^{1}$ Performance on this task was surprisingly low. When the target was present, subjects picked the correct person on only about $70 \%$ of occasions. When the target was absent, subjects nevertheless chose someone on roughly $30 \%$ of occasions, despite knowing that half the arrays would not contain the target.

This level of performance is particularly striking, because the arrays used by Bruce et al. (1999) were constructed in some dimensions to optimize performance. All images were taken in good lighting, from very similar fullface poses, and all were taken on the same day, eliminating transient differences in (for example) hairstyle, weight, or age. However, they were taken with different cameras, leading to different superficial image qualities. This has led some researchers (Hancock, Bruce, \& Burton, 2000) to suggest that the task is carried out by subjects in a rather unsophisticated "image matching" fashion and that the change in camera can make this match difficult.

The difficulty of matching can also be observed in settings with different task demands. For example, when the Bruce et al. (1999) array task is reduced to a 10-alternative forced choice, so that all arrays contain the target, and subjects know this, performance still remains surprisingly low, at 79\% (Bruce, Henderson, Newman, \& Burton, 2001). In

Correspondence should be addressed to A. M. Burton, Department of Psychology, University of Glasgow, Glasgow G12 8QQ, Scotland (e-mail: mike@psy.gla.ac.uk).

Note-This article was accepted by the previous editorial team, when Colin M. MacLeod was Editor. fact, the low levels of performance persist, even when subjects are shown two images and have to decide whether or not they are the same person (Henderson, Bruce, \& Burton, 2001). Kemp, Towell, and Pike (1997) showed that people find it very difficult to match a passport-type photo to a real person, reporting very high error rates for supermarket checkout staff attempting to verify store cards bearing a photograph of the carrier. They concluded that security would not be enhanced by the introduction of such cards.

This poor performance for unfamiliar faces stands in contrast to our very good performance with familiar faces. For example, viewers familiar with targets can recognize them trivially in arrays such as shown in Figures 1 and 2 and can even do so with high accuracy in severely degraded images, such as those provided by poor-quality security surveillance cameras (Burton, Wilson, Cowan, \& Bruce, 1999; Liu, Seetzen, Burton, \& Chaudhuri, 2003).

Here, we are primarily interested in unfamiliar-face processing and, particularly, individual differences in a facematching task. Although performance with unfamiliar faces is generally poor, there is nevertheless quite considerable variation among subjects. In the first experiment we report below, using the Bruce et al. (1999) matching task, performance ranged between $50 \%$ and $96 \%$. It therefore seems reasonable to ask what factors might predict this performance.

There has been some previous research on individual differences in face recognition, although this has generally addressed the issue of face memory rather than face matching. Across a set of experiments involving a total of 400 participants, Woodhead and Baddeley (1981) showed recognition memory for previously unfamiliar faces to be very variable, ranging from $d^{\prime}$ of -0.5 to 6.8 in their sample. This study showed that recognition memory for faces correlated positively with recognition memory for other objects. Facial recognition memory has also been shown to correlate positively with processing speed in infants (Rose, Feldman, \& Jankowski, 2003) and with percep- 


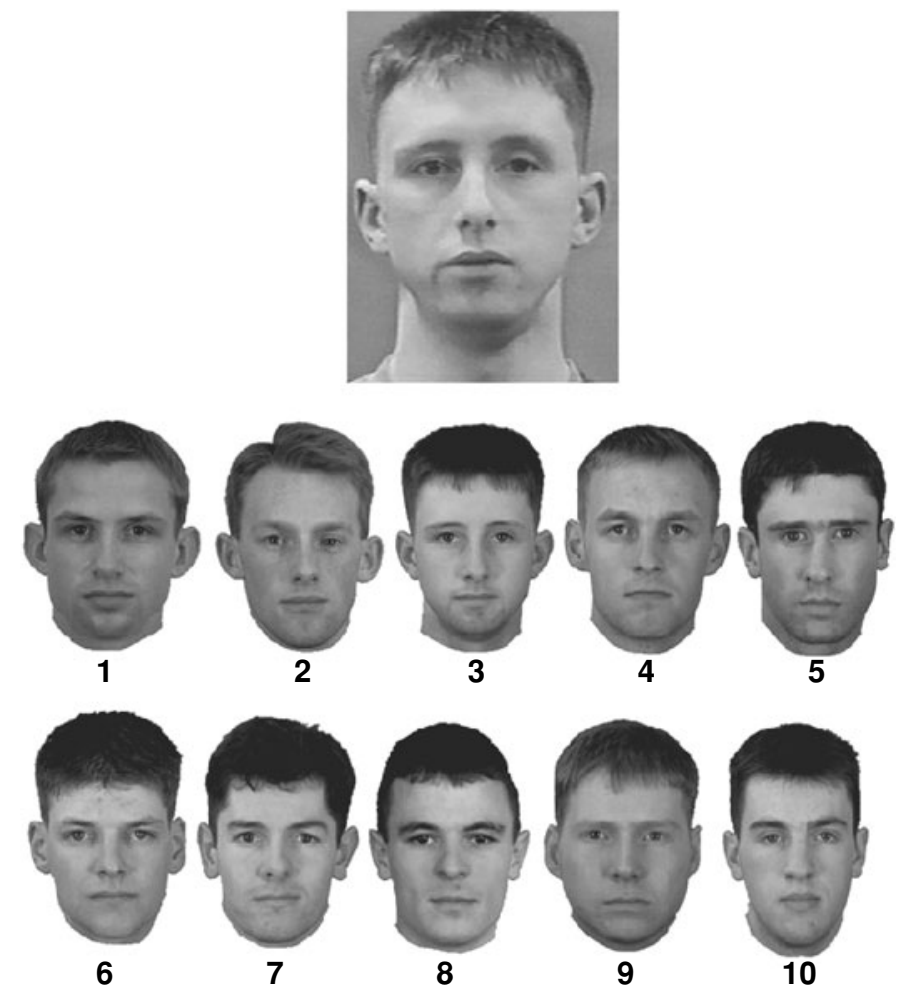

Figure 1. The face of the person shown at the top may or may not be one of the 10 faces below it. The subjects' task was to decide whether the target face was present among the 10 faces below it and, if so, which one it was. Note-From "Verification of Face Identities From Images Captured on Video," by V. Bruce et al., 1999, Journal of Experimental Psychology: Applied, 5, p. 344. Copyright 1999 by the American Psychological Association. Reprinted with permission.

tual speed in 11-year-old children in a longitudinal study (Rose \& Feldman, 1995).

The search for further predictors of face memory has produced inconclusive results. Mueller, Bailis, and Goldstein (1979) found that anxiety predicted false positives (FPs), but not hits, in a recognition memory procedure. However, Nowicki, Winograd, and Millard (1979) found anxiety to predict hits (negatively) but to be uncorrelated with FPs. This inconsistency is also apparent for field dependence. Witkin, Dyk, Faterson, Goodenough, and Karp (1974) predicted that field dependents would be more accurate in face recognition than would field independents, since they are giving more attention to the social content of their surroundings. Some studies have supported this prediction (Messick \& Damarin, 1964), others have shown the precise converse pattern (Lavrakas, Buri, \& Mayzner, 1976), and others have found no relationship between field dependence and face recognition performance (Courtois \& Mueller, 1982; Ryan \& Schooler, 1998).

These studies, rather inconclusive as they are, all addressed face memory. The literature contains very few reports analyzing individual differences in face matching. Schretlen, Pearlson, Anthony, and Yates (2001) found that performance of normal adults on the Benton Facial Recognition Test correlated positively with perceptual speed and total cerebral volume. Alexander et al. (1999) asked subjects to match photographs of frontal faces to two photographs of faces in different views. They found that individual differences in PET activation of the general object perception and attention system predicted the accuracy of matching unfamiliar faces.

In this study, we applied a small battery of tests to the subjects and asked what predicts performance on unfamiliar face matching. In later studies, we will be particularly interested in the associations between unfamiliar face matching and other face tasks (e.g., the same task upside down, or the same task with familiar faces). However, we begin with a set of standard tests, including perceptual speed and field dependence. If we can find a set of measures that reliably predicts subjects' performance in this task, then this might plausibly be useful in forensic situations. For example, choice of witness for particular identification tasks might be informed by such results. Of more theoretical interest, it may be possible to understand the fundamental processes involved in face matching, if standard tests can be shown to predict different aspects of performance. 


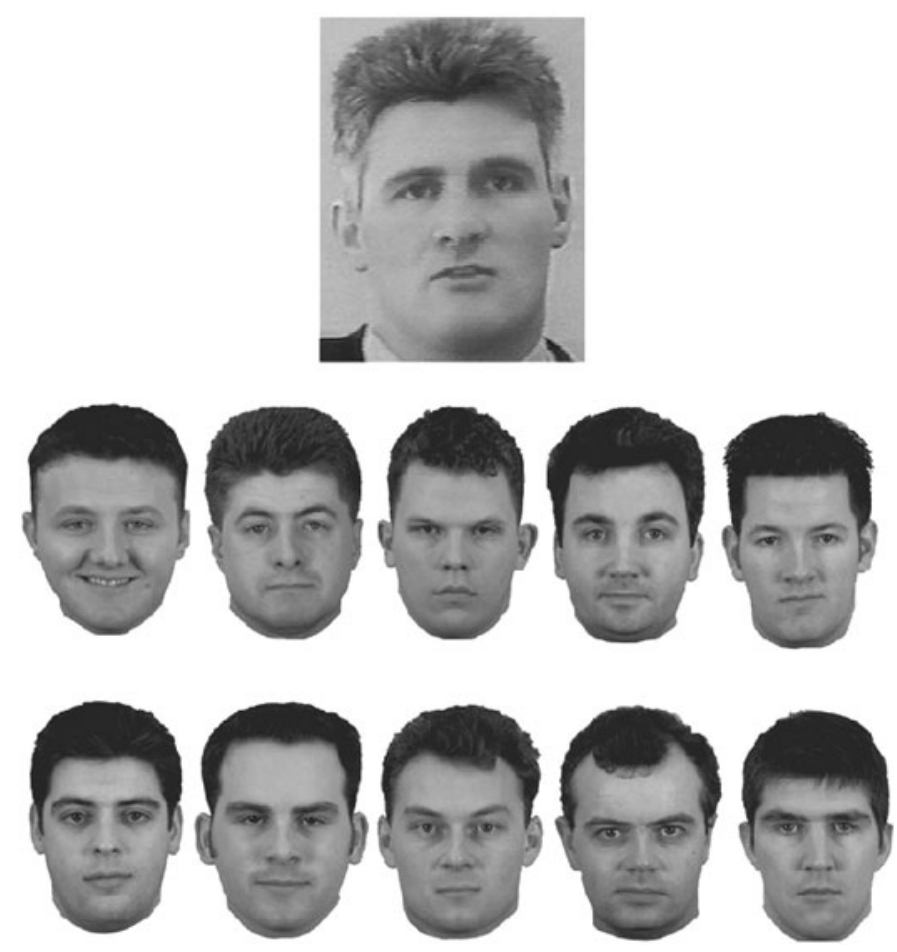

Figure 2. The face of the person shown at the top may or may not be one of the 10 faces below it. The subjects' task was to decide whether the target face was present among the 10 faces below it and, if so, which one it was. Note-From "Verification of Face Identities From Images Captured on Video," by V. Bruce et al., 1999, Journal of Experimental Psychology: Applied, 5, p. 347. Copyright 1999 by the American Psychological Association. Reprinted with permission.

\section{EXPERIMENT 1}

In this experiment, we examined performance on Bruce et al.'s (1999) face matching task and its covariation with matching other objects, visual short-term memory, visual differentiation, and perceptual speed.

\section{Method}

Subjects. Thirty students from the University of Glasgow participated in the experiment. They ranged in age from 18 to 32 years. There were 14 men and 16 women. All subjects had normal or corrected-tonormal vision.

Procedure and Stimuli. The subjects were tested individually in a session lasting approximately $90 \mathrm{~min}$. All completed the following tests.

Matching unfamiliar faces. One hundred sixty matching arrays were used, as illustrated in Figures 1 and 2. These were, in fact, the same arrays as used by Bruce et al. (1999). Each stimulus showed a still image, taken from a high-quality video camera, and showed a full-face view in neutral expression. Below this, there were 10 images of faces, each a full-face photograph in neutral expression, taken with a studio camera. All images were shown in gray scale, and the size of each face image was approximately $7 \times 10 \mathrm{~cm}$. Stimuli were presented in a large booklet, one array ( 1 video still +10 photos) per page. These images were taken from the UK Home Office PITO database, and all showed young, clean-shaven Caucasian men. All images had been taken on the same day; individuals showed little variation in hairstyle, face shape, and so on.
In half of the arrays, the target person (video still) was also present among the 10 face photographs. These arrays were constructed such that the 9 distractors were those faces rated most like the target in a prior similarity-ratings study. For each target (video still), a targetabsent array was also constructed, such that the 10 distractor images were those judged most like the target in a previous study. Full details of this database and construction of the arrays can be found in Bruce et al. (1999).

Each subject completed 80 trials: 40 target-present trials and 40 target-absent trials, intermixed in a random order. Two sets of stimuli were constructed, and presentation was counterbalanced across the experiment, such that each target face was seen in a target-present array by half the subjects and in a target-absent array by the remaining subjects.

The subjects' task was to match the identity of the target in the video-still image to the lineup photographs of faces. They were instructed that the target might or might not be present in each array. If they decided that it was present, they should write its number in the answer sheet, and, otherwise, they should mark an X. There was no time limit for this task, and the subjects were encouraged to perform as accurately as possible.

Visual short term memory. Forty-five line drawings of objects were used as stimuli. All pictures were presented in black and white. They belonged to different classes of object, including foods, clothes, instruments, animals, plants, birds, fruits, fish, transport, insects, and furniture. Pictures were formed into six sets, containing (in steps) between 5 and 10 objects. For each set, objects were arranged in a circular display on a single sheet of paper and were chosen so that each set contained objects from several categories. 
The procedure following this task was very simple, as described by Miller (1956). The subjects were shown each of the six sets of objects for $3 \mathrm{sec}$, starting with the set containing the fewest items. They were asked to recall as many stimuli as possible for each set. The measure of performance was the total number of stimuli that could be remembered across trials.

Group Embedded Figures Test. Field dependence was measured by the Group Embedded Figures Test (GEFT), which requires subjects to separate an item from the field in which it is incorporated (Witkin et al., 1974). The test consisted of 8 target simple geometric figures and 18 complicated geometric figures split into two sets. The subjects' task was to find the specific simple figure embedded into the complex figures. A maximum of 5 min was allowed for each set, and the measure of performance was accuracy.

Perceptual Speed Test. The Perceptual Speed Test was taken from the Kit of Factor-Referenced Cognitive Tests (see Ekstrom, French, \& Harman, 1979). This has three subtests. In the Finding A's Test, subjects are shown sets of words and must find as many $A$ s as possible within 2 min. In the Number Comparison Test, subjects are shown pairs of multidigit numbers and must classify these as the same or different. Scores reflect the number of correct classifications made within 2 min. In the Identical Pictures Test, subjects are required to match a target line-drawn figure to an array of five variants. Scores reflect the number of correct matches within $90 \mathrm{sec}$.

Matching Familiar Figures Test. The Matching Familiar Figures Test (MFFT) is a common measure for the cognitive style of impulsivity versus reflexivity (Kagan, 1965). The test consists of 20 standard line drawings of common objects (targets) and six variants of each object. The subjects' task is to find the variant that identically matches the target object.

Recognition memory for unfamiliar face pictures. Thirty-five images of unfamiliar faces were used as stimuli. These were taken from a database different from that used for the face matching test. All images were of young, clean-shaven, Caucasian males, and image software was used to remove background and clothing. Images were all presented in gray scale.

Fifteen of these images were presented to the subjects for $5 \mathrm{sec}$ each. They were told that they would subsequently be asked to pick out these same images from a larger set of faces. The Perceptual Speed tests (which involve no face stimuli) were then administered before the test phase. At test, the subjects were shown the original 15 images intermixed with the remaining 20 images. Images were shown one at a time, and the subjects were asked to decide, for each image, whether it had been seen in the learning phase.

\section{Results}

Table 1 shows the mean performance on each measure. In this and subsequent analyses, we have broken down the face matching data as follows. First, we show overall accuracy (i.e., the number of trials on which the subjects gave a correct response). This measure therefore combined hits (correctly picking the target in target-present trials) and correct rejections on target-absent trials. We also provide a more detailed breakdown of the data. For target-present trials, we report hits, misses (i.e., falsely responding that the target was absent), and misidentifications (choosing the wrong face as a match). For target-absent trials, we show FPs (i.e., incorrectly picking a target). Data for the face image recognition memory test is similarly broken down into hits, misses, and FPs.

Table 2 (top section) shows the relationship between performance on the face matching task and the subjects' performance on the psychometric variables. The first column shows correlations with overall accuracy, and the
Table 1

Summary Data for All Measures in Experiment 1

\begin{tabular}{crr}
\hline Measure & $M$ & $S D$ \\
\hline Face Matching & & \\
Accuracy $(/ 80)$ & 65.9 & 9.7 \\
Hit $(/ 40)$ & 35.3 & 3.8 \\
Miss (/40) & 3.2 & 3.1 \\
MisID (/40) & 1.5 & 2.1 \\
FP (/40) & 9.3 & 8.6 \\
Other Tests & & \\
Visual STM (/45) & 27.8 & 4.3 \\
GEFT (/18) & 14.0 & 3.9 \\
Perceptual speed & & \\
Finding A's Test (/100) & 62.4 & 12.5 \\
Number Comparison (/48) & 35.0 & 6.6 \\
Identical Picture Test (/96) & 67.5 & 12.2 \\
MFFT (/20) & 13.3 & 4.2 \\
Face Image Memory & & \\
Accuracy (/35) & 28.3 & 3.2 \\
Hit (/15) & 11.6 & 2.0 \\
Miss (/15) & 3.3 & 2.0 \\
FP (/20) & 3.2 & 2.3 \\
\hline
\end{tabular}

Note-MisID, misidentification; FP, false positive; STM, short-term memory; GEFT, Group Embedded Figures Test; MFFT, Matching Familiar Faces Test.

remaining columns show correlations with the submeasures of face matching performance. The final row in this section relates the subjects' performance on face matching and face image recognition memory. Correlations shown are between corresponding scores (i.e., correlations of accuracy in the two measures, hits in the two measures, etc.).

Since different components of the accuracy scores appear to correlate differentially with the other measures, we also conducted intercorrelations within the five different measures of face matching. These are shown in Table 3.

\section{Discussion}

Summary scores. We note that the overall scores on the face matching task replicate results reported by Bruce et al. (1999). Out of 80 arrays, the subjects scored on average about $66(82 \%)$ correct. Furthermore, there were quite significant individual differences, a large standard deviation, and a range of $50 \%-96 \%$ correct on this task. This further emphasizes this strange finding: Given no time pressure and good-quality images taken in good lighting, from the same viewpoint, on the same day, the subjects nevertheless found it surprisingly hard to match faces of unfamiliar people. In fact, the subjects seemed to find it particularly difficult to decide that a face was not present. In the 40 target-present arrays, the subjects picked the correct face on average 35 times (i.e., $88 \%$ of the time). However, in the 40 target-absent arrays, the subjects were correct on average only 31 times ( $77 \%$ of the time).

The remaining variables provide good overall scores for examining individual differences in face matching performance. There were no obvious ceiling or floor effects in the psychometric variables, and reasonably large standard deviations suggest that it is worthwhile to try to establish whether there were systematic relationships between these variables and the face matching performance of interest. 
Table 2

Pearson's Correlation Between Performance on Matching Upright Unfamiliar Faces and Other Tasks Across Experiments

\begin{tabular}{|c|c|c|c|c|c|}
\hline \multirow[b]{2}{*}{ Task } & \multicolumn{5}{|c|}{ Matching Upright Unfamiliar Faces } \\
\hline & Accuracy & Hit & Miss & MisID & FP \\
\hline \multicolumn{6}{|l|}{ Experiment 1} \\
\hline Visual STM & $.493^{* *}$ & $.485^{* *}$ & -.277 & $-.436^{*}$ & -.346 \\
\hline GEFT & .172 & .085 & -.011 & -.131 & -.157 \\
\hline Perceptual speed: Finding A’s & $.455^{*}$ & $.597^{* *}$ & -.350 & $-.523^{* *}$ & -.254 \\
\hline Perceptual speed: Number comparison & .359 & .124 & -.043 & -.154 & -.352 \\
\hline Perceptual speed: Identical pictures & $.649^{* *}$ & .302 & -.121 & -.348 & $-.603^{* *}$ \\
\hline MFFT & $.528^{* *}$ & $.487^{* *}$ & $-.362^{*}$ & -.315 & $-.385^{*}$ \\
\hline Recognition memory for face images & $.442^{*}$ & $.429^{*}$ & $.382^{*}$ & - & $.381^{*}$ \\
\hline \multicolumn{6}{|l|}{ Experiment 2} \\
\hline Unfamiliar faces (lineup task, target inverted) & $.818^{* *}$ & $.764^{* *}$ & $.817^{* *}$ & $.717^{* *}$ & $.855^{* *}$ \\
\hline \multicolumn{6}{|l|}{ Experiment 3} \\
\hline Unfamiliar faces (lineup task, all faces inverted) & $.730^{* *}$ & $.739^{* *}$ & $.785^{* *}$ & $.537^{* *}$ & $.712^{* *}$ \\
\hline \multicolumn{6}{|l|}{ Experiment 4} \\
\hline $\begin{array}{l}\text { Unfamiliar faces (match/mismatch task, } \\
\text { both faces inverted) }\end{array}$ & $.772^{* *}$ & $.514^{* *}$ & - & - & $.598^{* *}$ \\
\hline \multicolumn{6}{|l|}{ Experiment 5} \\
\hline Unfamiliar faces (upright, match from memory) & $.820^{* *}$ & $.713^{* *}$ & $.768^{* *}$ & $.623^{* *}$ & $.694^{* *}$ \\
\hline Familiar face decision (upright, accuracy) & .010 & -.020 & .076 & -.051 & -.038 \\
\hline Familiar face decision (inverted, accuracy) & $.612^{* *}$ & $.483^{* *}$ & -.110 & $-.524^{* *}$ & $-.571^{* *}$ \\
\hline Familiar face decision (upright RT) & -.292 & -.336 & .314 & .123 & .169 \\
\hline Familiar face decision (inverted RT) & -.088 & -.009 & -.071 & -.084 & -.143 \\
\hline \multicolumn{6}{|l|}{ Experiment 6} \\
\hline Familiarized faces (upright) & .277 & .356 & .328 & .353 & .103 \\
\hline Familiarized faces (inverted) & $.673^{* *}$ & $.666^{*}$ & $.741^{* *}$ & $.526^{* *}$ & .732 ** \\
\hline
\end{tabular}

Note-For Experiments 2-6, values refer to associations of the same measure across tasks (i.e., accuracy with accuracy, hits with hits, etc.). MisID, misidentification; FP, false positive; STM, short-term memory; GEFT, Group Embedded Figures Test; MFFT, Matching Familiar Faces Test; RT, response time. $\quad{ }^{*} p<.05 . \quad{ }^{* *} p<.01$.

Individual differences in matching unfamiliar faces. As we noted above, there were large performance variations among our subjects on the unfamiliar face matching task. It seems from some of these results that this variation can be predicted to some extent by other visual tests.

First, we note that the GEFT provided no significant associations with measures of face matching. Using face memory tests, this task has sometimes predicted performance and has sometimes not (Courtois \& Mueller, 1981; Lavrakas et al., 1976; Ryan \& Schooler, 1998). Using face matching, there is no suggestion of an effect.

The visual short-term memory (STM) measure did provide a significant association with face matching. This was most pronounced for the overall accuracy measure but appeared to be carried by significant associations with hits and misidentifications. A possibly more interesting pattern of effects arises from measures of perceptual speed. Schretlen et al. (2001) found that perceptual speed could significantly predict individual differences in matching unfamiliar faces on the Benton test. That test uses images taken with the same camera but at different angles, whereas our replication here used same viewpoint but different cameras. Interestingly, the three measures showed quite different patterns. The Finding A's task correlates with overall accuracy and seems to mimic visual short-term memory, in that this appears to be carried by significant associations with hit and misidentification rate. The number comparison, on the other hand, showed no reliable associations. Finally, the Identical Pictures task again correlated highly with overall accuracy, but the effect seems to have been carried by the FPs. Therefore, it seems that the Finding A's task and the Identical Pictures task are picking up different aspects of the unfamiliar face matching results. The Finding A's task is associated only with performance on the target-present stimuli, whereas the Identical Pictures task is associated only with performance on the target-absent trials. In some ways, the Identical Figures task is most like the face matching task in format: Both require a match to target for simultaneously presented material. However, in the Identical Pictures task, there is always a target present, and so subjects do not have to make the present/absent judgment that is a key feature of the face matching task.

The MFFT shows a different pattern of data. As with other variables, there is a good correlation with overall accuracy, and this is carried by hits but also by misses and

Table 3

Pearson's Correlations Between the Face Matching Measures in Experiment 1

\begin{tabular}{ccccc}
\hline Matching Measure & Accuracy & Hit & Miss & MisID \\
\hline Hit & $.480^{* *}$ & & & \\
Miss & -.053 & $-.819^{* *}$ & & \\
MisID & $-.756^{* *}$ & $-.536^{* *}$ & -.046 & \\
FP & $-.923^{* *}$ & -.105 & -.299 & $.622^{* *}$ \\
\hline
\end{tabular}
Note $-N=30$. MisID, misidentification; FP, false positive. ${ }^{* *} p<$ .01 . 
FPs. So, this is the only psychometric variable producing a reliable association with miss scores.

The association between face matching and face image recognition memory is relatively straightforward, although comparatively modest. The three subcomponents of each test are associated significantly (hits with hits, misses with misses, and FPs with FPs), but not highly.

Intercorrelations between the different array measures. The patterns of association among the submeasures of face matching are also very interesting in themselves (Table 3). For example, note that hits and false positives do not correlate. Since FPs are the inverse of performance in the target-absent trials, this may be surprising. In effect, there is no relationship between subjects' ability to perform in the target-present and target-absent arrays. This is, at first sight, puzzling. However, it has considerable precedent in the face recognition literature. Using recognition memory tasks for unfamiliar faces, rather than simultaneous matching, a number of independent groups have reported the lack of correlation between hits and false positives (Bruce, Burton, \& Dench, 1994; Hancock, Burton, \& Bruce, 1996; Lewis \& Johnston, 1997; Vokey \& Read, 1992). This has led to considerable theorizing about the independence of different processes in face recognition and the different patterns of similarity and distinctiveness among them. We will return to a brief discussion of these findings again in the General Discussion section. However, the main purpose of this article is to ask what measures predict performance on unfamiliar face matching overall, and so we will not consider in depth those aspects of the data showing correlations among the subcomponents of this general accuracy.

\section{EXPERIMENT 2}

In Experiment 1, we found that subjects who were good at matching unfamiliar faces were also good at matching objects. Across a range of measures, we have gained moderately good predictive scores from general measures of visual processing onto the processing of unfamiliar faces, although there seem to be some interesting patterns of data, such that different aspects of visual processing (as measured by the different tasks) are picking up different components of the face matching task. Furthermore, these measures are predictive at least to the same level as one measure of face processing, recognition memory for face pictures. In Experiment 2, we examined the relation between face matching and inverted face matching.

There is good evidence in the literature that inverted faces are not processed for identity in the same manner as are upright faces. Faces suffer considerable performance deficits, across a number of recognition measures, when inverted. It has been suggested that this might reflect a disruption of the processes normally engaged in face recognition-particularly, configural processing. Several authors have suggested that inverted faces are therefore processed in a manner more similar to the general object processing system than to the normal face recognition system (de
Gelder \& Rouw, 2000; Farah, Wilson, Drain, \& Tanaka, 1995; Haxby et al., 1999; Moscovitch, Winocur, \& Behrmann, 1997). One reason for this suggestion is that there is a large literature showing no evidence for an association between subjects' identification performance on upright and inverted familiar faces (for a review, see Valentine, 1988), suggesting that the two tasks rely on different processes. In Experiment 2, we therefore asked, what is the relation between upright and inverted processing in this matching task using unfamiliar faces?

\section{Method}

Subjects. Thirty paid University of Glasgow students (9 males, 21 females) participated in the experiment, ranging in age from 17 to 23 years. All had normal or corrected-to-normal vision.

Stimuli and Procedure. The face matching arrays from Experiment 1 were used in this experiment. The arrays were divided into two sets, such that, in half, the target face (i.e., the still from the video) was presented upside down. The 10 photographic images in each array were always presented in the correct orientation.

Each subject completed 40 matching trials with the target face oriented correctly and 40 trials with the target oriented upside down. In half the arrays, the target was present, and in half, the target was absent. The subjects were informed of this and asked to decide for each array whether the target was present and, if so, to indicate the correct match. The order in which the subjects performed the match (upside down or correct orientation) was counterbalanced across the experiment. As in Experiment 1, the procedure was self-paced, with no time pressure, and the subjects were requested to be as accurate as possible.

\section{Results and Discussion}

Mean accuracy for the upright and inverted matching tasks was 31.0 and 26.0 (out of 40 ), respectively $(S D=5.5$ and 6.2 , respectively) $[t(29)=7.67, p<.01]$. In fact, the subjects were significantly poorer in the inverted condition than in the upright condition for every subcomponent of the accuracy score. ${ }^{2}$ Correlations between performance in upright and inverted versions of the task are shown in Table 2 (i.e., correlations of accuracy with accuracy, hits with hits, etc).

These data provide a further replication of the findings of Bruce et al. (1999) and of Experiment 1, in that the subjects found the standard upright match of unfamiliar faces surprisingly difficult (overall performance level of $77 \%$ in this case). The inverted matches were somewhat harder (overall performance of 65\%). More surprising, the results of this experiment showed very high positive correlations between matching upright and inverted unfamiliar faces in all five lineup measures. It appears that the best predictor of the unfamiliar face matching task was performance on a version of the same test in which the target face was inverted. This is rather a striking finding, since it has generally been held that matching inverted faces engages superficial processes, rather than the sophisticated configural processes normally held to be the exclusive characteristic of upright face processing. This is the first hint in these data that rather unsophisticated processes may be taking place in upright unfamiliar face matching. This is a hypothesis put forward previously 
by Bruce et al. (1999) and Hancock et al. (2000). These authors proposed that unfamiliar face matching may be better thought of as simple image matching, rather than involving any face-specific processes.

The face processing literature generally does not show any evidence for an association between upright and inverted face recognition (see Valentine, 1988). In fact, in some experiments, a negative correlation has been observed (Yin, 1969). Although this negative association is not always found (e.g., Flin, 1985), positive correlations are never reported. However, previous research on face inversion has generally used a face memory task of some sort. This may provide the explanation for the difference between the findings of the present experiment and the more usual findings. Furthermore, tests of face memory have often used the same image of each person at learning and test phases, just as we did in the recognition memory test in Experiment 1. Image matching (as opposed to image memory) is a very simple task, and arrays of the sort in Figure 1 are solved trivially if the target image is one of the same images as the 10 test faces. This observation begins to suggest that there may be important aspects of unfamiliar face processing that are missed in studies deploying the same images throughout.

If our hypothesis is correct, and the processes engaged in unfamiliar face matching are (at least to some extent) simple image matching, then we should be able to observe associations between normal upright matching and more difficult matching tasks with the same stimuli. We explored this is in the next experiment.

\section{EXPERIMENT 3}

In this experiment, we aimed to replicate Experiment 2, but with a more demanding image matching task. Here, we examined the association between upright unfamiliar face matching and inverted face matching in which both the target face and the 10 faces for matching were presented upside down.

\section{Method}

Subjects. Thirty University of Glasgow undergraduate students (7 males, 21 females) participated in the experiment, ranging in age from 18 to 23 years. All had normal or corrected-to-normal vision. None of the subjects had taken part in the previous experiments. They either were paid a small sum for participation or received course credit.

Stimuli and Procedure. The stimuli and procedure were identical to those of Experiment 2, with the sole exception that, here, the inverted face matching trials involved a match from an inverted target to 10 inverted faces. Again, order of presentation (upright or inverted) was counterbalanced across subjects .

\section{Results and Discussion}

Mean accuracy for the upright and inverted matching tasks was 27.1 and 18.9 (out of 40 ), respectively [ $S D=$ 6.9 and 5.1, respectively; $t(29)=9.53, p<.01$ ]. Again, the subjects were significantly poorer in the inverted than the upright condition for every subcomponent of the accu- racy score. Correlations between performance in upright and inverted versions of the task are shown in Table 2.

As with Experiment 2, there were very high associations between matching unfamiliar faces on this array task and completing the same task with the entire display inverted. It seems that, to some considerable extent, the same processes were being employed in both tasks. It is relatively uncontentious to claim that the upside-down version of this task is not employing the configural processing usually thought to be a hallmark of face processing. However, if this is the case, then it seems to follow that these processes cannot be being engaged in the upright unfamiliar face matching task.

Of course, these data do not allow us to conclude that unfamiliar face matching is only image matching. If this were the case, then we would expect the overall performance measures for the upright and inverted versions of this task to be the same. However, they were not. In Experiments 2 and 3, the task was performed significantly more accurately for upright faces than for inverted faces. We will return to this difference in the General Discussion section. In the following experiment, we asked whether the effects we have observed so far are tied to the specific 1-in-10 lineup task.

\section{EXPERIMENT 4}

Experiments 2 and 3 showed very high associations between matching performance upright and inverted for a particular task. However, this is not the most commonly used matching task in the face recognition literature. Instead, a simple same/different task is often used, in which only two images are shown at once, and subjects must decide whether these are the same or different people. There are a number of reasons why such a task might, in principle, differ from the 1-in-10 task used here. In particular, the presence of the distractors might encourage a particular mode of processing, not normally employed. To check this, we next repeated the comparison of upright and inverted matching using a simple same/different task.

\section{Method}

Subjects. Thirty students ( 16 females and 14 males) participated in the experiment either for money or for credit hours. The subjects ranged in age from 18 to 22 years. All had normal or corrected-tonormal vision, and none had taken part in the previous experiments.

Stimuli and Procedure. Pairs of images were used as stimuli. Each pair consisted of one high-quality video still image (of the form shown as the target in Figure 1) and one high-quality photograph (of the form shown in the 10-member arrays in Figure 1). These images came from the same database as used to construct stimuli for Experiments 1-3. One hundred twenty matching pairs and 120 mismatching pairs were constructed, such that mismatching pairs were similar in appearance. Images were cropped as described above and were presented in gray scale at a size of $5 \times 7 \mathrm{~cm}$.

Each subject was presented with 120 trials during the experiment and was asked to respond "same" or "different" using a keypress. Half the trials were presented upright, and half were inverted (both faces). Furthermore, half the pairs showed the same person, and half showed a different person. Match-mismatch items, and upright- 
inverted items were counterbalanced across the experiment, such that each stimulus item appeared equally often in each trial type. Upright and inverted stimuli were intermixed during the experiment, in a different random order for each subject. Stimuli were presented on a computer screen, and the experiment was self-paced.

\section{Results and Discussion}

Mean accuracy for the upright and inverted matching tasks was 47.9 and 40.8 (out of 60 ), respectively [ $S D=$ 5.8 and 6.7 , respectively; $t(29)=8.95, p<.01]$. The subjects performed significantly better in the upright condition than in the inverted condition for both hits $[24.5 / 30$ vs. $22.3 / 30 ; t(29)=3.1, p<.01)$ and FPs $[6.6 / 30$ vs. $11.5 / 30 ; t(29)=6.4, p<.01]$. Correlations between performance in upright and inverted versions of the task are shown in Table 2 (note that this task did not give rise to independent miss or misidentification errors).

The results of this experiment were very consistent with those of Experiments 2 and 3. Again, we found a rather low level of performance in matching, even in this simple match/mismatch task. Note that in the upright version of the match, for the target-present trials, the subjects still managed to score only $24.5 / 30$ correct ( $82 \%$ ). In this condition, there were no distractors present at all, and to perform correctly, the subjects simply had to confirm that these were two images of the same person. The fact that they could not do this more accurately is consistent with previous work on face matching and carries forensic implications.

Theoretically, the most interesting effect is that there were very high levels of correlation between upright and inverted conditions, suggesting that similar processes underlie the two tasks. It seems, then, that the correspondence between upright and inverted unfamiliar face processing is robust across matching tasks and is not an artifact of the 1-in-10 lineup task used so far. In the next experiment, we examined the relation between familiar and unfamiliar face matching.

\section{EXPERIMENT 5}

In Experiments 2, 3, and 4, we found very high positive correlations between matching upright and matching inverted unfamiliar faces. We have tentatively suggested that these high correlations might have been due to the fact that unfamiliar faces are not engaging the processes normally engaged by familiar faces. In order to examine this possibility further in the present experiment, we tested the subjects' performance with highly familiar face matching and unfamiliar face matching.

One of the difficulties in comparing familiar and unfamiliar face processing is that familiar face processing is so robust. People are able to recognize the faces of those familiar to them in a very wide range of viewing conditions, even in very severely degraded visual environments, such as security CCTV (Burton, Wilson, et al., 1999). Given this, it is not possible directly to compare an array task such as we have used for unfamiliar faces with the equivalent stimulus constructed with highly familiar faces. In such a situation, subjects score at ceiling levels of performance, meaning that one cannot look for associations between performance on familiar face matching and other variables.

To deal with this, in this experiment, we examined the subjects' ability to make a simple personal decision to a familiar (famous) face. We chose a nationality decision, for a set of faces all of whom are either British or American. We examined accuracy (which we expected to be high) and response time (RT) for making this decision for familiar faces and the relationship between familiar and unfamiliar face matching tasks. Furthermore, we presented the familiar faces upside down, in order to examine any possible relationship between upright unfamiliar face matching and inverted familiar face recognition.

In this experiment, we also introduced a further manipulation with the unfamiliar face processing task. Since previous research with unfamiliar faces has tended to use memory tasks (for clear forensic reasons), we have here introduced an immediate memory version of the array task.

\section{Method}

Subjects. Thirty paid University of Glasgow undergraduate students ( 10 males, 20 females) participated in the experiment, ranging in age from 17 to 25 years. All had normal or corrected-to-normal vision, and all were British. None had taken part in the previous experiments.

Stimuli and Procedure. The subjects were tested individually in a session of approximately $40 \mathrm{~min}$. The arrays for the matching task were the same as those used in Experiment 1. These were divided into two sets such that, for each subject, half the arrays were presented simultaneously (target +10 photographs) and the remaining half were presented sequentially. Sets were counterbalanced such that, across the experiment, each target face occurred equally often in a simultaneous array and a sequential array.

Procedure for the matching task was as described in the previous experiments. For the sequential, immediate memory, version of the task, each trial consisted of a single target face, presented for $5 \mathrm{sec}$, followed by a $5-\mathrm{sec}$ gap, followed by presentation of the 10-photo array. The subjects were again asked to decide whether or not the target was present and, if so, to identify him in the 10-photo array. There was no time limit, and the subjects were encouraged to be as accurate as possible.

For the famous face tasks, a set of 80 celebrity faces were collected: Half were British, and half were American. All of the famous faces were presented in gray scale and were of Caucasian males, but with variations in age, hairstyle, expressions, lighting conditions, and viewing angles. For display on a computer screen, these were scaled to roughly $10 \times 7 \mathrm{~cm}$. The faces were split into two groups: Half were presented upright, and half were inverted. The stimuli were counterbalanced, such that, across the experiment, all faces occurred equally often upright and inverted. Recognition of the celebrity faces was tested using a semantic task and was run on a G3 Macintosh computer using Superlab Pro software. Each face was presented on the screen until the subjects responded, and there was a 1-sec interstimulus interval. The subjects' task was to classify each face as American or British, by pressing one of two labeled response keys. They were instructed to respond as fast and as accurately as possible. The orientation of stimuli was mixed during the presentation, with independent randomization of the order of stimuli for each subject.

\section{Results}

Results for the unfamiliar face processing task showed mean accuracy for matching and memory of 28.0 and 
26.1 (out of 40), respectively $[S D=4.95$ and 5.08 , respectively; $t(29)=3.5, p<.01]$. Correlations between performance in matching and memory versions of the task are shown in Table 2. For the familiar face task (nationality decision), mean accuracy was 37.1 and 27.4 for the upright and inverted conditions, respectively $[S D=1.3$ and 3.9 , respectively; $t(29)=13.8, p<.01]$. The advantage for upright faces was also reflected in the RT data, with mean correct RTs of 1,303 and $1,792 \mathrm{msec}$, respectively $[S D=243$ and 398, respectively; $t(29)=6.7, p<.01]$.

More interesting were the correlation scores with familiar face processing. There was no reliable correlation observed between upright and inverted famous faces for either the accuracy or the RT measures $(r \mathrm{~s}=.178$ and .287 , respectively). Table 2 shows the associations between unfamiliar face matching and the four measures of familiar face recognition (upright, inverted, accuracy, and RT). ${ }^{3}$

\section{Discussion}

First, we note from these data that unfamiliar face memory, even measured on an immediate memory test, is more difficult than is unfamiliar face matching. However, even on the matching version of the task, we have replicated the, by now, standard finding that subjects are remarkably inaccurate at matching these images, scoring at only $71 \%$ accurate overall on this occasion. The correlations between the two versions of this task were very high (Table 2), with matching being predicted well by memory on the same task.

Next, we note that the familiar faces chosen were well known by these subjects, as evident from the high levels of performance in the nationality classification. We have replicated the well-known inversion effect, such that the subjects were severely impaired on a recognition task by turning the faces upside down. Interestingly, and consistent with the literature on familiar face processing, the results show that there was no reliable association between the subjects' performance on the upright and inverted versions of this familiar face processing task, in either accuracy or RT measures. This is in very great contrast to the consistent correlations between upright and inverted processing shown for unfamiliar faces (Experiments 2, 3, and 4). This seems to offer suggestive evidence that the processing of identity in familiar and unfamiliar faces is qualitatively different, a position to which we will return in the General Discussion section.

Finally, we examine the associations between familiar and unfamiliar face processing, as shown in Table 2. Consider the famous face accuracy data first. There was no evidence of an association between unfamiliar face processing and upright familiar face processing (and this was true regardless of whether a matching or memory task was used for measuring performance on unfamiliar faces; see Note 3). Of course, it is difficult to interpret these data, since there was a ceiling effect in the upright face classification task (as we hoped, since we wanted to choose faces that the subjects would know). However, there was a very striking, high level of association between the unfamiliar face matching tasks and the inverted famous face classification. These high levels of association strongly suggest that the processing of upright unfamiliar faces is closely related to the processing of inverted famous faces. Since we found no evidence for an association between upright and inverted famous face classification, this again suggests that there is a qualitative difference between processing familiar and unfamiliar faces. In contrast to accuracy, RT data for the familiar face tasks did not provide any reliable associations with the measures of unfamiliar face processing, and so we will not consider this measure further.

Although these data are potentially interesting, there are some problems in interpreting them. We propose that the strong association between inverted famous faces and upright unfamiliar faces is a novel and important result. This correlation is particularly interesting since recognition tasks with familiar faces have never revealed a positive correlation between upright and inverted versions (see Valentine, 1988). However, the other half of the task in Experiment 5, using upright faces, was less easy to interpret, since there were clear ceiling effects. In a final experiment, we attempted to pursue the issue of familiar and unfamiliar face processing further, using a familiarization procedure. Our intention was to provide an experiment in which tasks for familiar and unfamiliar faces could be equated, without giving rise to ceiling or floor effects.

\section{EXPERIMENT 6 Unfamiliar and Familiarized Faces}

The target images, at the top of the arrays illustrated in Figure 1, were stills from a high-quality video. The original source videos from this database comprised 30-sec clips of each person moving his head left-right, up-down, and nonrigidly through talking (though no sound was present). This gave us the opportunity to familiarize the subjects with the target people before showing the matching arrays. This is a manipulation that, despite the relatively brief exposure, has been shown to improve subjects' matching ability significantly (Bruce et al., 2001). In this experiment, we presented the subjects with 1-in-10 lineup arrays, for half of which they had been familiarized with the target. We examined their performance on unfamiliar arrays, as in all the experiments above, and related this to performance on upright and inverted familiarized face matching. This allowed us to compare familiar and unfamiliar faces on the same task and (through counterbalancing of stimuli) across the same faces.

\section{Method}

Subjects. Sixty paid University of Glasgow undergraduate students ( 23 males, 37 females) participated in the experiment, ranging in age from 17 to 27 years. All had normal or corrected-to-normal vision. None had taken part in the previous experiments.

Stimuli and Procedure. All subjects were tested on 80 arrays, constructed in the same fashion as described for Experiment 1. For half the arrays, we had familiarized the subjects with the target by 
showing them video clips of each of the 40 target faces. These clips showed rigid and nonrigid motion but were presented without sound. During this learning phase, the subjects were instructed to try to learn the faces, and were told that they would later be asked to recognize the people depicted. Each clip was $30 \mathrm{sec}$ long, and the clips were shown one after another, with 1-sec gaps between them. Across the experiment, target faces were counterbalanced in such a way that each was used equally often as a learned and an unfamiliar face.

At test, the subjects saw familiarized and unfamiliar arrays blocked, with order counterbalanced across the experiment. For the familiarized block, half the subjects saw the arrays upright and half saw them inverted. The unfamiliar arrays were always presented upright. As in the previous experiments, within each condition, half the test arrays had the target present and half had the target absent. The subjects were instructed that this would be the case and were asked, for each array, to say whether the target was present and, if so, to indicate the matching face.

\section{Results and Discussion}

For the subjects tested on upright familiarized faces, there was a significant advantage in matching familiarized faces over unfamiliar faces [mean familiarized $=$ $35 / 40$, mean unfamiliar $=29.6 / 40 ; S D=3.4$ and 4.3, respectively; $t(29)=6.21, p<.01]$. For the subjects tested on inverted familiarized faces, performance was better on the (upright) unfamiliars [mean familiarized $=$ 27.0/40, mean unfamiliar $=30.9 / 40 ; S D=6.1$ and 6.2, respectively; $t(29)=4.34, p<.01]$. Correlations between performance on unfamiliar faces and familiarized faces (upright and inverted) are shown in Table 2.

These results show a simple pattern. First, the familiarization procedure was successful, to some extent, in teaching the subjects the identities. Mean accuracy increased from 29.6 to 35 out of 40 items correct, even though the familiarization procedure was relatively brief (30 sec per face). Table 2 demonstrates that once these faces have become familiar, performance on the matching task does not correlate with performance on the same task, with unfamiliar faces. Note that items were rotated around conditions across the experiment; thus, the same faces were used equally often as familiar and unfamiliar items. The only difference between the conditions was the level of familiarity to the subjects. Furthermore, this absence of correlation was not due to a ceiling effect, as was the case in the previous experiment. The rather short familiarization phase appears to have been sufficient to change the way in which the faces were matched but was not sufficient to reach ceiling levels of performance.

Results for the subjects who were tested on the familiar faces upside down show an equally interesting pattern. The overall performance level on these faces was reduced, as one would expect. However, performance on these familiarized faces correlated highly with performance on the same task for unfamiliar upright faces (Table 2, final row). This pattern of results provides the clearest evidence so far for our tentative hypothesis outlined above: Processes involved in unfamiliar face matching show a strong association with those recruited for inverted familiar face matching. However, using the same task, familiar and unfamiliar face processing appear to dissociate when both are tested in normal (upright) conditions.

\section{GENERAL DISCUSSION}

We have presented six experiments addressing unfamiliar face matching. Our initial question was, What predicts performance on a 1 -in-10 face matching task? Experiment 1 showed that one can find reliable predictors from some standard tests of perception and memory. Significant, although relatively modest, correlations were found with perceptual speed, visual STM, and figure matching. Interestingly, these variables predicted different parts of the matching data, with some predicting accuracy in target-present arrays and others predicting accuracy in target-absent arrays. This is consistent with the lack of correlation between performance on target-present and target-absent arrays. This absence of correlation is not the focus of the present article, although it is interesting in its own right and worthy of further investigation.

Experiments 2, 3, and 4 showed that the face identity matching task is predicted very strongly by performance on the same task using inverted faces. This is of particular interest since traditional studies with familiar face identification never show a positive association between recognition performance upright and inverted. Experiments 5 and 6 showed that familiar face recognition was rather poorly associated with the unfamiliar face matching task, unless the familiar faces were inverted. This was shown using very familiar faces (Experiment 5), in which a completely different task (semantic decision) for inverted famous faces was strongly associated with matching unfamiliar faces - a surprising result given the absence of such associations in the recognition literature. In Experiment 6, we were able to compare familiar and unfamiliar faces on the same task and to demonstrate that rather little exposure is required before previously unfamiliar faces become familiar, at which stage matching performance ceases to correlate with performance on unfamiliar faces. However, consistent with the hypothesis that familiar faces recruit some processes that are not involved in unfamiliar faces or inverted faces, a strong correlation remained between upright unfamiliar and inverted familiar faces.

We take these results to imply that unfamiliar face matching is qualitatively different from familiar face recognition. The strong correlation between upright and inverted versions of the same unfamiliar face task suggests that similar processes are at play. This association is not found for familiar faces (Valentine, 1988), a finding reproduced (rather weakly) in our Experiment 5 and more convincingly in Experiment 6. Face inversion effects are generally attributed to the notion that inverted faces cannot be processed configurally, a key component of normal face recognition (e.g., Bartlett \& Searcy, 1993; Freire, Lee, \& Symons, 2000; Tanaka \& Sengco, 1997). If this is true, then the present findings suggest that unfamiliar faces in general do not support configural processing. Without such processing, we have to ask how the task is possible at all.

One extreme solution to this problem would be to assert that unfamiliar faces are processed only as patterns and are matched in the same way as any other visual stimu- 
lus, without recourse to any information about faces in general (Hancock et al., 2000). However, this is not a completely satisfactory explanation for our data, because Experiments 2, 3, and 4 demonstrated that unfamiliar face matching is more difficult when one or all of the faces are inverted. There are very high levels of association between performance upright and inverted, but there is a straightforward advantage in matching the upright versions. Since the same visual information is present in all the displays, the advantage for upright recognition must reflect some processing at a more sophisticated level than simple pattern matching. In fact, Sekuler, Gaspar, Gold, and Bennett (2004) have suggested that inversion effects are due to quantitative rather than qualitative differences in processing. These researchers used a visual discrimination approach and unfamiliar faces only. They found that observers use the same regions of a face to make visual discriminations, regardless of orientation. It seems that our results are quite consistent with this position. If subjects are somehow matching particular areas of a face ("features") in both cases, then general face knowledge may help in the upright case. However, this leaves open to question whether the inversion effect for familiar faces reflects the same operations as does the inversion effect for unfamiliar faces.

In attempting to address these questions, it is important to note that the inversion effects we have reported here relate specifically to matching or recognition tasks (i.e., those in which the computation of individual identity is necessary). It is certainly the case that face inversion effects vary according to the perceiver's task (e.g., Bartlett \& Searcy, 1993; Enns \& Shore, 1997; Sergent, 1984). For tasks other than identification or matching, familiar and unfamiliar faces may well be processed similarly and similarly across orientations. We should be clear that, in proposing that unfamiliar faces are processed in ways qualitatively different from familiar faces, the argument refers to computation of identity only.

Of course, there is a very large literature that highlights differences between familiar and unfamiliar face processing, and these differences were the focus of considerable early work in the field (e.g., Bruce, 1986; Klatzky \& Forrest, 1984; Young, Hay, McWeeny, Flude, \& Ellis, 1985). Indeed, a dissociation between unfamiliar face matching and familiar face recognition has long been established in the neuropsychological literature (Malone, Morris, Hay, \& Levin, 1982; Young, Newcombe, de Haan, Small, \& Hay, 1993). Current theoretical work tends to focus on one or another type of face processing, and models of familiar face processing (Bruce \& Young, 1986; Burton, Bruce, \& Hancock, 1999) have little to say on the subject of unfamiliar faces. We suggest that this is rational in the light of the data.

Returning to our title, we suggest that unfamiliar faces are not processed (for identity) like faces, in exactly the same sense in which inverted faces are not processed like faces. Therefore, conflating familiar and unfamiliar faces into a single theory of face processing seems to be an unpromising approach. Having said this, it is obvious that unfamiliar faces become familiar all the time, as we come to know new people. Given the large processing differences between these two types of visual stimulus, development of a satisfactory account of face learning poses a significant challenge.

\section{REFERENCES}

Alexander, G. E., Mentis, M. J., Van Horn, J. D., Grady, C. L., Berman, K. F., Furey, M. L., Et Al. (1999). Individual differences in PET activation of object perception and attention systems predict face matching accuracy. NeuroReport, 10, 1965-1971.

Bartlett, J. C., \& Searcy, J. (1993). Inversion and configuration of faces. Cognitive Psychology, 25, 281-316.

BRuce, V. (1986). Influences of familiarity on the processing of faces. Perception, 15, 387-397.

Bruce, V., Burton, A. M., \& Dench, N. (1994). What's distinctive about a distinctive face? Quarterly Journal of Experimental Psychology, 47A, 119-141.

Bruce, V., Henderson, Z., Greenwood, K., Hancock, P. J .B., BurTON, A. M., \& Miller, P. (1999). Verification of face identities from images captured on video. Journal of Experimental Psychology: Applied, 5, 339-360.

Bruce, V., Henderson, Z., Newman, C., \& Burton, A. M. (2001). Matching identities of familiar and unfamiliar faces caught on CCTV images. Journal of Experimental Psychology: Applied, 7, 207-218.

BRuce, V., \& Young, A. W. (1986). Understanding face recognition. British Journal of Psychology, 77, 305-327.

Burton, A. M., Bruce, V., \& Hancock, P. J. B. (1999). From pixels to people: A model of familiar face recognition. Cognitive Science, 23, 1-31.

Burton, A. M., Wilson, S., Cowan, M., \& Bruce, V. (1999). Face recognition in poor-quality video: Evidence from security surveillance. Psychological Science, 10, 243-248.

Courtois, M. R., \& Mueller, J. H. (1981). Target and distractor typicality in facial recognition. Journal of Applied Psychology, 66, 639-645.

DE Gelder, B., \& Rouw, R. (2000). Paradoxical configuration effects for faces and objects in prosopagnosia. Neuropsychologia, 38, 12711279 .

Ekstrom, R. B., French, J. W., \& Harman, H. H. (1979). Cognitive factors: Their identification and replication. Multivariate Behavioral Research Monographs, 79, 3-84.

EnNS, J. T., \& ShORE, D. I. (1997). Separate influences of orientation and lighting in the inverted-face effect. Perception \& Psychophysics, 59, 23-31.

Farah, M. J., Wilson, K. D., Drain, H. M., \& Tanaka, J. R. (1995). The inverted faces inversion effect in prosopagnosia: Evidence for mandatory, face-specific perceptual mechanisms. Vision Research, 35, 2089-2093.

FLIN, R. H. (1985). Development of face recognition: An encoding switch? British Journal of Psychology, 76, 123-134.

Freire, A., LeE, K., \& Symons, L. A. (2000). The face-inversion effect as a deficit in the encoding of configural information: Direct evidence. Perception, 29, 159-170.

Hancock, P. J. B., Bruce, V., \& Burton, A. M. (2000). Recognition of unfamiliar faces. Trends in Cognitive Sciences, 4, 330-337.

Hancock, P. J. B., Burton, A. M., \& Bruce, V. (1996). Face processing: Human perception and principal components analysis. Memory \& Cognition, 24, 26-40.

Haxby, J. V., Ungerleider, L. G., Clark, V. P., Schouten, J. L., Hoffman, E. A., \& Martin, A. (1999). The effects of face inversion on activity in human neural system for face and object perception. Neuron, 22, 189-199.

Henderson, Z., Bruce, V., \& Burton, A. M. (2001). Matching the faces of robbers captured on video. Applied Cognitive Psychology, 15, 445-464.

KaGAN, J. (1965). Reflection-impulsivity and reading ability in primary grade children. Child Development, 36, 609-628.

Kemp, R., Towell, N., \& Pike, G. (1997). When seeing should not be believing: Photographs, credit cards and fraud. Applied Cognitive Psychology, 11, 211-222. 
Klatzky, R. L., \& Forrest, F. H. (1984). Recognizing familiar and unfamiliar faces. Memory \& Cognition, 12, 60-70.

Lavrakas, P. J., Buri, J. R., \& Mayzner, M. S. (1976). A perspective on the recognition of other-race faces. Perception \& Psychophysics, 20, 475-481.

Lewis, M. B., \& Johnston, R. A. (1997). Familiarity, target set, and false positives in face recognition. European Journal of Cognitive Psychology, 9, 437-459.

Liu, C. H., Seetzen, H., Burton, A. M., \& Chaudhuri, A. (2003). Face recognition is robust with incongruent image resolution: Relationship to security video images. Journal of Experimental Psychology: Applied, 9, 33-41.

Malone, D. R., Morris, H. H., Kay, M. C., \& Levin, H. S. (1982) Prosopagnosia: A double dissociation between the recognition of familiar and unfamiliar faces. Journal of Neurology, Neurosurgery, \& Psychiatry, 45, 820-822.

Messick, S., \& Damarin, F. (1964). Cognitive styles and memory for faces. Journal of Abnormal \& Social Psychology, 69, 313-318.

Miller, G. A. (1956). The magical number seven, plus or minus two: Some limits on our capacity for processing information. Psychological Review, 63, 81-97.

Moscovitch, M., Winocur, G., \& Behrmann, M. (1997). What is special about face recognition? Nineteen experiments on a person with visual object agnosia and dyslexia but normal face recognition. Journal of Cognitive Neuroscience, 9, 555-604.

Mueller, J. H., Bailis, K. L., \& Goldstein, A. G. (1979). Depth of processing and anxiety in facial recognition. British Journal of Psychology, 70, 511-515.

Nowicki, S., Winograd, E., \& Millard, B. A. (1979). Memory for faces: A social learning analysis. Journal of Research in Personality, 13, 460-468.

Rose, S. A., \& Feldman, J. F. (1995). Prediction of IQ and specific cognitive abilities at 11 years from infancy measures. Developmental Psychology, 31, 685-696.

Rose, S. A., Feldman, J. F., \& Jankowski, J. J. (2003). Infant visual recognition memory: Independent contributions of speed and attention. Developmental Psychology, 39, 563-571.

Ryan, R. S., \& Schooler, J. W. (1998). Whom do words hurt? Individual differences in susceptibility to verbal overshadowing. Applied Cognitive Psychology, 12, 105-125.

Schretlen, D. J., Pearlson, G. D., Anthony, J. C., \& Yates, K. O. (2001). Determinants of Benton Facial Recognition Test performance in normal adults. Neuropsychology, 15, 405-410.

Sekuler, A. B., Gaspar, C. M., Gold, J. M., \& Bennett, P. J. (2004). Inversion leads to quantitative, not qualitative, changes in face processing. Current Biology, 14, 391-396.
Sergent, J. (1984). An investigation into component and configural processes underlying face recognition. British Journal of Psychology, 75, 221-242.

Tanaka, J. W., \& Sengco, J. A. (1997). Features and their configuration in face recognition. Memory \& Cognition, 25, 583-592.

VAlentine, T. (1988). Upside-down faces: A review of the effect of inversion upon face recognition. British Journal of Psychology, 79, 471-491.

VoKey, J. R., \& READ, J. D. (1992). Familiarity, memorability, and the effect of typicality on the recognition of faces. Memory \& Cognition, 20, 291-302.

Witkin, H. A., Dyk, R. B., Faterson, H. F., Goodenough, D. R., \& KARP, S. A. (1974). Psychological differentiation: Studies of development (2nd ed.). New York: Wiley.

Woodhead, M. M., \& BadDEley, A. D. (1981). Individual differences and memory for faces, pictures, and words. Memory \& Cognition, 9, 368-370.

YIN, R. K. (1969). Looking at upside-down faces. Journal of Experimental Psychology, 81, 141-145.

Young, A. W., Hay, D. C., McWeeny, K. H., Flude, B. M., \& Ellis, A. W. (1985). Matching familiar and unfamiliar faces on internal and external features. Perception, 14, 737-746.

Young, A. W., Newcombe, F., De Haan, E. H. F., Small, M., \& Hay, D. C. (1993). Face perception after brain injury: Selective impairments affecting identity and expression. Brain, 116, 941-959.

\section{NOTES}

1. Answers: In Figure 1, the target face is number 3; in Figure 2, the target face is not present in the array.

2 . Because of the very large amount of data generated by these experiments, we have presented only a summary here. In particular, we have not presented performance measures separately for hits, misses, misidentifications, and FPs or for correlations between these. The data are available from the authors on request.

3 . For reasons of economy, we have not shown the correlations between unfamiliar face memory and the familiar face processing task. In fact, these showed exactly the same pattern as the associations between unfamiliar face matching and the familiar face task (i.e., there were significant correlations between inverted familiar face accuracy and unfamiliar face memory scores on accuracy, hits, misidentifications, and FPs. All other associations were nonsignificant. Details are available from the authors.

(Manuscript received April 14, 2004; revision accepted for publication May 16, 2005.) 